PROCEEDINGS OF THE

AMERICAN MATHEMATICAL SOCIETY

Volume 125, Number 12, December 1997, Pages 3695-3703

S $0002-9939(97) 04111-7$

\title{
A SINGULAR INTEGRAL OPERATOR WITH ROUGH KERNEL
}

\author{
DASHAN FAN AND YIBIAO PAN
}

(Communicated by J. Marshall Ash)

\begin{abstract}
Let $b(y)$ be a bounded radial function and $\Omega\left(y^{\prime}\right)$ an $H^{1}$ function on the unit sphere satisfying the mean zero property. Under certain growth conditions on $\Phi(t)$, we prove that the singular integral operator

$$
T_{\Phi, b} f(x)=\text { p.v. } \int_{\mathbb{R}^{n}} f\left(x-\Phi(|y|) y^{\prime}\right) b(y)|y|^{-n} \Omega\left(y^{\prime}\right) d y
$$

is bounded in $L^{p}\left(\mathbb{R}^{n}\right)$ for $1<p<\infty$.
\end{abstract}

\section{INTRODUCTION}

Let $\mathbb{R}^{n}, n \geq 2$, be the $n$-dimensional Euclidean space and $S^{n-1}$ be the unit sphere in $\mathbb{R}^{n}$ equipped with normalized Lebesgue measure $d \sigma=d \sigma\left(x^{\prime}\right)$. Let $\Omega(x)|x|^{-n}$ be a homogeneous function of degree $-n$, with $\Omega \in L^{1}\left(S^{n-1}\right)$ and

$$
\int_{S^{n-1}} \Omega\left(x^{\prime}\right) d \sigma\left(x^{\prime}\right)=0
$$

where $x^{\prime}=x /|x|$ for any $x \neq 0$.

Suppose that $b(t)$ is an $L^{\infty}$ function on $(0, \infty)$ and $\Phi(t)$ is a real-valued smooth function on $(0, \infty)$ satisfying

$$
\begin{gathered}
|\Phi(t)| \leq C_{1}|t|^{d}, \quad\left|\Phi^{\prime \prime}(t)\right| \leq C_{2}|t|^{d-2}, \\
C_{3}|t|^{d-1} \geq\left|\Phi^{\prime}(t)\right| \geq C_{4}|t|^{d-1},
\end{gathered}
$$

for some $d \neq 0$ and $t \in(0, \infty)$, where $C_{1}, C_{2}, C_{3}$ and $C_{4}$ are positive constants independent of $t$. A simple example of such $\Phi$ is $\Phi(t)=t$. by

For given $\Phi(t), b(t)$ and $\Omega\left(y^{\prime}\right)$, we define the singular integral operator $T_{\Phi, b}(f)$

$$
T_{\Phi, b}(f)(x)=\text { p.v. } \int_{\mathbb{R}^{n}} K(y) f\left(x-\Phi(|y|) y^{\prime}\right) d y,
$$

where $y^{\prime}=y /|y| \in S^{n-1}, K(y)=b(|y|) \Omega\left(y^{\prime}\right)|y|^{-n}$ and $f \in \mathcal{S}\left(\mathbb{R}^{n}\right)$.

For the sake of simplicity, we denote $T_{\Phi, b}=T_{b}$ if $\Phi(t)=t$ and $T_{\Phi, b}=T$ if $\Phi(t)=t$ and $b(t) \equiv 1$.

The investigation of the operators $T_{b}$ began with Calderón-Zygmund's pioneering study of the operator $T$ (see Theorem A below). The operator $T_{b}$, whose kernel

Received by the editors October 25, 1995 and, in revised form, August 11, 1996.

1991 Mathematics Subject Classification. Primary 42B20.

Key words and phrases. Singular integral, rough kernel, Hardy space.

The second author was supported in part by a grant from the National Science Foundation.

(C) 1997 American Mathematical Society 
has the additional roughness in the radial direction due to the presence of $b$, was first studied by R. Fefferman ([Fe]) and subsequently by many other authors ([Ch], [DR], [Fa], [FP2], [Na]). We list some of the known results below.

Theorem A ([CZ2], also see [SW]). If $\Omega \in L \log ^{+} L$, then the operator $T$ is bounded in $L^{p}\left(\mathbb{R}^{n}\right)$ for $1<p<\infty$.

Theorem B ([Ch], [Na], see also [DR]). If $\Omega \in L^{q}\left(S^{n-1}\right)$ for some $q>1$, then the operator $T_{b}$ is bounded in $L^{p}\left(\mathbb{R}^{n}\right)$ space for $1<p<\infty$.

Theorem $\mathbf{C}([\mathrm{Fa}])$. If $\Omega \in H^{1}\left(S^{n-1}\right)$, then $T_{b}$ is bounded in $L^{2}\left(\mathbb{R}^{n}\right)$.

The space $H^{1}\left(S^{n-1}\right)$ in Theorem $\mathrm{C}$ represents the Hardy space on the unit sphere, whose definition will be reviewed in section 2 . In order to make comparisons among the conditions imposed on $\Omega$ in Theorems A-C, we point out that on $S^{n-1}$, for any $q>1, L^{q} \subseteq L \log ^{+} L \subseteq H^{1}\left(S^{n-1}\right)$ and all inclusions are proper.

In both $T$ and $T_{b}$, the singularity is along the diagonal $\{x=y\}$. Recently many problems in analysis have led one to consider singular integrals with singularity along more general sets, some in the form of $\{x=\Psi(y)\}$ (see [St]). Here we focus our attention on singular integrals $T_{\Phi, b}$ which have singularity along sets of the form $\left\{x=\Phi(|y|) y^{\prime}\right\}$. We are able to obtain the $L^{p}$ boundedness of $T_{\Phi, b}$ under the assumption $\Omega \in H^{1}\left(S^{n-1}\right)$. We state our result as follows:

Theorem D. Let $T_{\Phi, b}$ be the singular integral operator defined by (1.4). If $\Omega \in$ $H^{1}\left(S^{n-1}\right)$ and satisfies (1.1), then $T_{\Phi, b}$ is a bounded operator in $L^{p}\left(\mathbb{R}^{n}\right)$ for $1<$ $p<\infty$.

By the relationship among $L^{q}\left(S^{n-1}\right), L \log ^{+} L\left(S^{n-1}\right)$, and $H^{1}\left(S^{n-1}\right)$ displayed above, one sees that, even in the special case $\Phi(t) \equiv t\left(T_{\Phi, b}=T_{b}\right)$, Theorem $\mathrm{D}$ represents an improvement over previous results. The method we use to prove Theorem $\mathrm{D}$ is quite flexible and can be used to prove the $L^{p}$ boundedness of $T_{\Phi, b}$ under a weaker condition on $b(\cdot)$ than $b \in L^{\infty}$ (see [DR], [Fa], and the remark at the end of this article).

Throughout this paper, the letter $C$ will denote a positive constant that may vary at each occurrence but is independent of the essential variables.

\section{Definitions AND LEMMAS}

Recall that the Poisson kernel on $S^{n-1}$ is defined by

$$
P_{r y^{\prime}}\left(x^{\prime}\right)=\left(1-r^{2}\right) /\left|r y^{\prime}-x^{\prime}\right|^{n},
$$

where $0 \leq r<1$ and $x^{\prime}, y^{\prime} \in S^{n-1}$.

For any $f \in \mathcal{S}^{\prime}\left(S^{n-1}\right)$, we define the radial maximum function $P^{+} f\left(x^{\prime}\right)$ by

$$
P^{+} f\left(x^{\prime}\right)=\sup _{0 \leq r<1}\left|\int_{S^{n-1}} f\left(y^{\prime}\right) P_{r x^{\prime}}\left(y^{\prime}\right) d \sigma\left(y^{\prime}\right)\right|,
$$

where $\mathcal{S}^{\prime}\left(S^{n-1}\right)$ is the space of Schwartz distributions on $S^{n-1}$. The Hardy space $H^{1}\left(S^{n-1}\right)$ is the linear space of distributions $f \in \mathcal{S}^{\prime}\left(S^{n-1}\right)$ with the finite norm $\|f\|_{H^{1}\left(S^{n-1}\right)}=\left\|P^{+} f\right\|_{L^{1}\left(S^{n-1}\right)}<\infty$. The space $H^{1}\left(S^{n-1}\right)$ was well-studied in [Co] (see also [CTW]). In particular, it was shown that $H^{1}\left(S^{n-1}\right)$ has the atomic decomposition property, which will be reviewed below. 
An exceptional atom is an $L^{\infty}$ function $E(x)$ satisfying $\|E\|_{\infty} \leq 1$. A regular $q$-atom is an $L^{q}(1<q \leq \infty)$ function $a(\cdot)$ that satisfies

$$
\begin{gathered}
\operatorname{supp}(a) \subset S^{n-1} \cap\left\{y \in \mathbb{R}^{n}:|y-\zeta|<\rho \text { for some } \zeta \in S^{n-1} \text { and } \rho \in(0,1]\right\} ; \\
\int_{S^{n-1}} a\left(\xi^{\prime}\right) d \sigma\left(\xi^{\prime}\right)=0, \\
\|a\|_{q} \leq \rho^{(n-1)(1 / q-1)} .
\end{gathered}
$$

A $q$-block is an $L^{q}(1<q \leq \infty)$ function that satisfies the above conditions $(2.1)$ and (2.3).

From [Co] or [CTW], we find that any $\Omega \in H^{1}\left(S^{n-1}\right)$ has an atomic decomposition $\Omega=\sum \lambda_{j} a_{j}$, where the $a_{j}$ 's are either exceptional atoms or regular $q$-atoms and $\sum\left|\lambda_{j}\right| \leq C\|\Omega\|_{H^{1}\left(S^{n-1}\right)}$. In particular, if $\Omega \in H^{1}\left(S^{n-1}\right)$ has the mean zero property (1.1), then all the atoms $a_{j}$ in the atomic decomposition can be chosen to be regular $q$-atoms for a fixed $q, 1<q \leq \infty$.

In the rest of this paper, for any non-zero $\xi=\left(\xi_{1}, \xi_{2}, \ldots, \xi_{n}\right) \in \mathbb{R}^{n}$, we write $\xi /|\xi|=\xi^{\prime}=\left(\xi_{1}^{\prime}, \xi_{2}^{\prime}, \ldots, \xi_{n}^{\prime}\right)=\left(\zeta_{1}, \zeta_{2}, \ldots, \zeta_{n}\right)=\zeta$. Thus $\zeta \in S^{n-1}$. Also we use $\zeta_{*}$ to denote $\left(\zeta_{2}, \ldots, \zeta_{n}\right)$ and use $\xi_{*}$ to denote $\left(\xi_{2}, \ldots, \xi_{n}\right)$.

Suppose $n \geq 3$ and $a(\cdot)$ is an $\infty$-atom on $S^{n-1}$ with $\operatorname{supp}(a) \subseteq S^{n-1} \cap B(\zeta, \rho)$, where $B(\zeta, \rho)$ is the ball in $\mathbb{R}^{n}$ centered at $\zeta$. Let

$$
\begin{aligned}
& F_{a}\left(s, \xi^{\prime}\right)=\left(1-s^{2}\right)^{(n-3) / 2} \chi_{(-1,1)}(s) \int_{S^{n-2}} a\left(s,\left(1-s^{2}\right)^{1 / 2} \tilde{y}\right) d \sigma(\tilde{y}), \\
& G_{a}\left(s, \xi^{\prime}\right)=\left(1-s^{2}\right)^{(n-3) / 2} \chi_{(-1,1)}(s) \int_{S^{n-2}}\left|a\left(s,\left(1-s^{2}\right)^{1 / 2} \tilde{y}\right)\right| d \sigma(\tilde{y}) .
\end{aligned}
$$

Then, we have the following estimates for $F_{a}$ and $G_{a}$ when $n \geq 3$.

Lemma 2.1. Up to a constant multiplier independent of $a(\cdot), F_{a}\left(s, \xi^{\prime}\right)$ is an $\infty$ atom on $\mathbb{R}$ and $G_{a}\left(s, \xi^{\prime}\right)$ is an $\infty$-block on $\mathbb{R}$. More precisely, there is a constant $C$ which is independent of $a(\cdot)$ such that

$$
\begin{gathered}
\operatorname{supp}\left(F_{a}\right) \subseteq\left(\xi_{1}^{\prime}-2 r\left(\xi^{\prime}\right), \xi_{1}^{\prime}+2 r\left(\xi^{\prime}\right)\right) \\
\operatorname{supp}\left(G_{a}\right) \subseteq\left(\xi_{1}^{\prime}-2 r\left(\xi^{\prime}\right), \xi_{1}^{\prime}+2 r\left(\xi^{\prime}\right)\right) ; \\
\left\|F_{a}\right\|_{\infty} \leq C / r\left(\xi^{\prime}\right) ; \quad\left\|G_{a}\right\|_{\infty} \leq C / r\left(\xi^{\prime}\right) ; \\
\int_{\mathbb{R}} F_{a}(s) d s=0,
\end{gathered}
$$

where $r\left(\xi^{\prime}\right)=|\xi|^{-1}\left|A_{\rho} \xi\right|$ and $A_{\rho} \xi=\left(\rho^{2} \xi_{1}, \rho \xi_{*}\right)$.

The functions $F_{a}$ and $G_{a}$ can be similarly defined in the case $n=2$. Suppose $n=2$ and $a(\cdot)$ is an $\infty$-atom on $S^{1}$ satisfying $(2.1)-(2.3)$. The center of the support of $a(\cdot)$ is $\xi^{\prime}=\zeta=\left(\zeta_{1}, \zeta_{2}\right) \in S^{1}$. Let

$$
\begin{aligned}
& f_{a}(s, \zeta)=\left(1-s^{2}\right)^{-1 / 2} \chi_{(-1,1)}(s)\left(a\left(s,\left(1-s^{2}\right)^{1 / 2}\right)+a\left(s,-\left(1-s^{2}\right)^{1 / 2}\right)\right), \\
& g_{a}(s, \zeta)=\left(1-s^{2}\right)^{-1 / 2} \chi_{(-1,1)}(s)\left(\left|a\left(s,\left(1-s^{2}\right)^{1 / 2}\right)\right|+\left|a\left(s,-\left(1-s^{2}\right)^{1 / 2}\right)\right|\right) .
\end{aligned}
$$

Similar to Lemma 2.1, we have

Lemma 2.2. Up to a constant multiplier independent of $a(\cdot), f_{a}(s, \zeta)$ is a q-atom on $\mathbb{R}$ and $g_{a}(s, \zeta)$ is a q-block on $\mathbb{R}$ whose support is the same as that of $f_{a}$, where $q$ is any fixed number in the interval $(1,2)$. The radius of their support is $r\left(\xi^{\prime}\right)=$ $|\xi|^{-1}\left\{\rho^{4} \xi_{1}^{2}+\rho^{2} \xi_{2}^{2}\right\}^{1 / 2}$, and the center of their support is $\xi_{1}^{\prime}$. 
Lemma 2.1 and Lemma 2.2 are essentially Proposition 2.5 in [FP1]. For the sake of completeness, we state the proofs of (2.4) and (2.5) for the function $G_{a}$.

If $\rho>1 / 4$, clearly $\operatorname{supp}\left(G_{a}\right) \subseteq(-1,1)$ and $\left\|G_{a}\right\|_{\infty} \leq C$. So we can assume $0<\rho \leq 1 / 4$. Let $\zeta=\left(\zeta_{1},\left(1-\zeta_{1}^{2}\right)^{1 / 2} \tilde{\zeta}\right)$ for some $\tilde{\zeta} \in S^{n-2}$. If $G_{a}(s) \neq 0$, then

$$
\left(s,\left(1-s^{2}\right)^{1 / 2} \tilde{y}\right) \in B(\zeta, \rho)
$$

for some $\tilde{y} \in S^{n-2}$. Therefore we have

$$
2 \zeta_{1} s+2\left(1-s^{2}\right)^{1 / 2}\left(1-\zeta_{1}^{2}\right)^{1 / 2}\langle\tilde{\zeta}, \tilde{y}\rangle \geq 2-\rho^{2}
$$

for some $\tilde{y} \in S^{n-2}$. Since $\langle\tilde{\zeta}, \tilde{y}\rangle \leq 1$, we obtain

$$
\left(s-\zeta_{1}\right)^{2}+\left|\left(1-s^{2}\right)^{1 / 2}-\left(1-\zeta_{1}^{2}\right)^{1 / 2}\right|^{2} \leq \rho^{2},
$$

which implies that

$$
\begin{gathered}
\left|s-\zeta_{1}\right| \leq \rho, \\
\left|\left(1-s^{2}\right)^{1 / 2}-\left(1-\zeta_{1}^{2}\right)^{1 / 2}\right| \leq \rho,
\end{gathered}
$$

and

$$
\left|s-\zeta_{1}\right| \leq 2|\xi|^{-1}\left|A_{\rho} \xi\right|
$$

Inequalities (2.8) and (2.9) follow from (2.7) trivially. To see (2.10) we shall consider the following two cases.

Case a: $\left|\zeta_{1}\right|>3 / 4$. Then by (2.8) and (2.9) we have

$$
\left|s+\zeta_{1}\right| \geq 2\left|\zeta_{1}\right|-\left|s-\zeta_{1}\right|>1
$$

and

$$
\begin{aligned}
\left|s-\zeta_{1}\right| & \leq\left|s^{2}-\zeta_{1}^{2}\right| \\
& =\left|\left(1-s^{2}\right)^{1 / 2}-\left(1-\zeta_{1}^{2}\right)^{1 / 2}\right|\left|2\left(1-\zeta_{1}^{2}\right)^{1 / 2}+\left(1-s^{2}\right)^{1 / 2}-\left(1-\zeta_{1}^{2}\right)^{1 / 2}\right| \\
& \leq \rho^{2}+2 \rho\left(1-\zeta_{1}^{2}\right)^{1 / 2}=\rho^{2}+2 \rho\left|\zeta_{*}\right| \leq 2|\xi|^{-1}\left|A_{\rho} \xi\right| .
\end{aligned}
$$

Case b: $\left|\zeta_{1}\right| \leq 3 / 4$. Then $1 / 2 \leq\left(1-\zeta_{1}^{2}\right)^{1 / 2}$. By $(2.8)$ we find

$$
\left|s-\zeta_{1}\right| \leq \rho<\rho^{2}+2 \rho\left(1-\zeta_{1}^{2}\right)^{1 / 2}=\rho^{2}+2 \rho\left|\zeta_{*}\right| \leq 2|\xi|^{-1}\left|A_{\rho} \xi\right|,
$$

which proves (2.10).

By letting $r\left(\xi^{\prime}\right)=|\xi|^{-1}\left|A_{\rho} \xi\right|$, we see that (2.4) is satisfied.

It remains to verify $(2.5)$ for $G_{a}$. To this end, we first assume that $\left(1-\zeta_{1}^{2}\right)^{1 / 2}=$ $\left|\zeta_{*}\right| \leq 99 \rho$. Then by $(2.3)$ we find

$$
\left\|G_{a}\right\|_{\infty} \leq(100 \rho)^{n-3} \omega_{n-2}\|a\|_{\infty} \leq 100^{n-3} \omega_{n-2} \rho^{-2} \leq C r\left(\xi^{\prime}\right)^{-1},
$$

where $C=100^{n-3} \omega_{n-2}$ and $\omega_{n-2}=\int_{S^{n-2}} d \sigma(\tilde{y})$.

Next we assume that $\left(1-\zeta_{1}^{2}\right)^{1 / 2}=\left|\zeta_{*}\right|>99 \rho$. By (2.9) we find

$$
\left(1-\zeta_{1}^{2}\right)^{1 / 2} / 2 \leq\left(1-s^{2}\right)^{1 / 2} \leq 2\left(1-\zeta_{1}^{2}\right)^{1 / 2} .
$$

For $\varepsilon>0$, let

$$
\Gamma(\varepsilon)=\left\{y \in \mathbb{R}^{n-1}: 1-\varepsilon \leq\langle y, \tilde{\zeta}\rangle \leq 1\right\} .
$$

When $\varepsilon$ is small we have

$$
\int_{\tilde{y} \in S^{n-2} \cap \Gamma(\varepsilon)} d \sigma(\tilde{y}) \cong \varepsilon^{(n-2) / 2} .
$$


By (2.1) we find

$$
\begin{aligned}
\{\tilde{y} \in & \left.S^{n-2}: a\left(s,\left(1-s^{2}\right)^{1 / 2} \tilde{y}\right) \neq 0\right\} \\
& \subseteq\left\{\tilde{y} \in S^{n-2}: 2 \zeta_{1} s+2\left(1-s^{2}\right)^{1 / 2}\left(1-\zeta_{1}^{2}\right)^{1 / 2}\langle\tilde{\zeta}, \tilde{y}\rangle \geq 2-\rho^{2}\right\} \\
& \subseteq\left\{\tilde{y} \in S^{n-2}: 1-\left(1-\zeta_{1}^{2}\right)^{-1 / 2}\left(1-s^{2}\right)^{-1 / 2} \rho^{2} / 2 \leq\langle\tilde{\zeta}, \tilde{y}\rangle \leq 1\right\}=\Gamma(\varepsilon),
\end{aligned}
$$

where $\varepsilon=\rho^{2} / 2\left(1-\zeta_{1}^{2}\right)^{-1 / 2}\left(1-s_{1}^{2}\right)^{-1 / 2}$. Thus by (2.11) we have

$$
\begin{aligned}
\left\|G_{a}\right\|_{\infty} & \leq 2^{n-3}\left(1-\zeta_{1}^{2}\right)^{(n-3) / 2} \rho^{-n+1} \int_{\tilde{y} \in S^{n-2} \cap \Gamma(\varepsilon)} d \sigma(\tilde{y}) \\
& \leq C\left(1-\zeta_{1}^{2}\right)^{(n-3) / 2} \rho^{-n+1} \rho^{n-2}\left(1-\zeta_{1}^{2}\right)^{(n-2) / 2} \\
& =C\left|\zeta_{*}\right|^{-1} \rho^{-1} \leq C r\left(\xi^{\prime}\right)^{-1} .
\end{aligned}
$$

This completes the proof of Lemma 2.1 for the function $G_{a}$.

Let $\Psi \in \mathcal{S}(\mathbb{R})$ such that $\widehat{\Psi}(t)=e^{-t^{2}}$ and define $\Psi_{k}$ on $\mathbb{R}^{n}$ by $\widehat{\Psi}_{k}(\xi)=$ $\widehat{\Psi}\left(2^{d k} r\left(\xi^{\prime}\right)|\xi|\right)$. Then we have the following

Lemma 2.3. The maximal operator $f \rightarrow \sup _{k}\left|\Psi_{k} * f\right|$ is bounded in $L^{p}\left(\mathbb{R}^{n}\right)$ for $1<p<\infty$.

Proof. By the definition of $\Psi_{k}$ we find

$$
\Psi_{k}(x)=2^{-d k} \rho^{-2} \Psi\left(x_{1} 2^{-d k} \rho^{-2}\right) \prod_{j=2}^{n}\left\{2^{-d k} \rho^{-1} \Psi\left(x_{j} 2^{-d k} \rho^{-1}\right)\right\} .
$$

Thus we have

$$
\sup _{k}\left|\Psi_{k} * f\right| \leq C M_{1} M_{2} \cdots M_{n}(f)
$$

where

$$
M_{j} f(x)=\sup _{h>0} \frac{1}{2 h} \int_{-h}^{h}\left|f\left(x_{1}, \ldots, x_{j-1}, x_{j}-u, x_{j+1}, \ldots, x_{n}\right)\right| d u .
$$

By the $L^{p}$ boundedness of the 1-dimensional Hardy-Littlewood maximal function, we obtain the $L^{p}$ boundedness of the operator $f \rightarrow \sup _{k}\left|\Psi_{k} * f\right|$. The lemma is proved.

\section{Proof of Theorem D}

We shall only prove the $L^{p}$ boundedness of $T_{\Phi, b}$ under the assumption that $\Phi$ satisfies (1.2) and (1.3) for some positive $d$. The argument for the case $d<0$ is similar and requires only minor modifications.

Noting that $T_{\Phi, b}(f)$ is equal to

$$
\int_{\mathbb{R}^{n}}|y|^{-n} b(|y|) \Omega\left(y^{\prime}\right) f\left(x-\Phi(|y|) y^{\prime}\right) d y,
$$

where $\Omega \in H^{1}\left(S^{n-1}\right)$ satisfies the mean zero property (1.1), we can write $\Omega=$ $\sum \lambda_{j} a_{j}$, where $\sum\left|\lambda_{j}\right| \leq C\|\Omega\|_{H^{1}\left(S^{n-1}\right)}$ and each $a_{j}$ is an $\infty$-atom. So

$$
\left\|T_{\Phi, b} f\right\|_{p} \leq C \sum\left|\lambda_{j}\right|\left\|B_{j}(f)\right\|_{p}
$$

where

$$
B_{j}(f)(x)=\int_{\mathbb{R}^{n}} b(|y|)|y|^{-n} a_{j}\left(y^{\prime}\right) f\left(x-\Phi(|y|) y^{\prime}\right) d y
$$


with $a_{j}$ being an $\infty$-atom. Therefore, it suffices to show

$$
\left\|B_{j}(f)\right\|_{p} \leq C\|f\|_{p},
$$

where $C$ is independent of the atoms $a_{j}(\cdot)$.

For simplicity in our argument, we denote $a_{j}(\cdot)$ by $a(\cdot)$ and $B_{j}(f)$ by $B(f)$. Also without loss of generality we may assume that $\operatorname{supp}(a)$ is the ball $B(\mathbf{1}, \rho) \cap S^{n-1}$, where $\mathbf{1}=(1,0, \ldots, 0)$. Let $I_{k}=\left(2^{k}, 2^{k+1}\right)$; then $B(f)(x)$ is equal to

$$
\int_{\mathbb{R}^{n}} b(|y|)|y|^{-n} a\left(y^{\prime}\right) \sum_{-\infty}^{\infty} \chi_{I_{k}}(|y|) f\left(x-\Phi(|y|) y^{\prime}\right) d y=\sum_{-\infty}^{\infty} \sigma_{k} * f(x),
$$

where

$$
\widehat{\sigma_{k}(\xi)}=\int_{2 k \leq|y| \leq 2^{k+1}} b(|y|)|y|^{-n} a\left(y^{\prime}\right) e^{-i \Phi(|y|)|\xi|\left\langle y^{\prime}, \xi^{\prime}\right\rangle} d y .
$$

Let

$$
\widehat{\mu_{k}(\xi)}=\int_{2^{k} \leq|y| \leq 2^{k+1}}|y|^{-n}\left|a\left(y^{\prime}\right)\right| e^{-i \Phi(|y|)|\xi|\left\langle y^{\prime}, \xi^{\prime}\right\rangle} d y
$$

and

$$
\sigma^{*} f(x)=\sup _{k}\left|\mu_{k} * f(x)\right| .
$$

Then we easily verify that $\left\|\hat{\mu}_{k}\right\|_{\infty}<C,\left\|\sigma_{k}\right\|_{1}<C$ uniformly for $k \in \mathbb{Z}$ and $\widehat{\sigma_{k}(0)}=0$ for all $k \in \mathbb{Z}$. The following lemma is an easy modification of Theorem $\mathrm{B}$ in $[\mathrm{DR}]$.

Lemma 3.1. Suppose that there exist $\alpha>0, \beta>0, d>0$ and $\rho>0$ such that

$$
\left|\widehat{\sigma_{k}(\xi)}\right| \leq C \min \left\{\left|2^{k d} A_{\rho} \xi\right|^{\alpha},\left|2^{k d} A_{\rho} \xi\right|^{-\beta}\right\}
$$

and suppose also that

$$
\left\|\sigma^{*} f\right\|_{p} \leq C\|f\|_{p}, \quad 1<p<\infty
$$

where $C>0$ is independent of $k \in \mathbb{Z}, \xi \in \mathbb{R}^{n}$ and $\rho>0$. Then the following two operators are bounded in $L^{p}\left(\mathbb{R}^{n}\right)$ for $1<p<\infty$ :

$$
B(f)=\sum_{k} \sigma_{k} * f, \quad g(f)=\left(\sum_{k}\left|\sigma_{k} * f\right|^{2}\right)^{1 / 2} .
$$

Also the bounds for these operators are independent of $\rho>0$.

We now verify that our $\sigma_{k}$ satisfies (i) in Lemma (3.1). We will only prove the case $n>2$, since the proof for $n=2$ is essentially the same (using Lemma 2.2 instead of Lemma 2.1).

For any $\xi \neq 0$, we choose a rotation $O$ such that $O(\xi)=|\xi| \mathbf{1}=|\xi|(1,0, \ldots, 0)$. Let $y^{\prime}=\left(s, y_{2}^{\prime}, y_{3}^{\prime}, \ldots, y_{n}^{\prime}\right)$. Then it is easy to see that

$$
\widehat{\sigma_{k}(\xi)}=\int_{I_{k}} b(t) t^{-1} \int_{S^{n-1}} a\left(O^{-1}\left(y^{\prime}\right)\right) e^{-i \Phi(t)|\xi|\left\langle 1, y^{\prime}\right\rangle} d \sigma\left(y^{\prime}\right) d t
$$

where $O^{-1}$ is the inverse of $O$. Now $a\left(O^{-1}\left(y^{\prime}\right)\right)$ is again an $\infty$-atom with support in $B(\zeta, \rho) \cap S^{n-1}$, where $\zeta=\xi^{\prime}$. Thus we have

$$
\widehat{\sigma_{k}(\xi)}=\int_{I_{k}} b(t) t^{-1} \int_{\mathbb{R}} F_{a}\left(s, \xi^{\prime}\right) e^{-i \Phi(t)|\xi| s} d s d t
$$


where $F_{a}\left(s, \xi^{\prime}\right)$ is the function defined in Lemma 2.1. By Lemma 2.1, without loss of generality we may assume that $F_{a}$ is a $q$-atom with support in $\left(-2 r\left(\xi^{\prime}\right), 2 r\left(\xi^{\prime}\right)\right)$ for $1<q<2$. Thus $A(s)=r\left(\xi^{\prime}\right) F_{a}\left(r\left(\xi^{\prime}\right) s, \xi^{\prime}\right)$ is a $q$-atom with support in the interval $(-1,1)$. After changing variables we have

$$
\widehat{\sigma_{k}(\xi)}=\int_{I_{k}} t^{-1} b(t) \int_{\mathbb{R}} A(s) e^{-i \Phi(t) r\left(\xi^{\prime}\right)|\xi| s} d s d t .
$$

So by the cancellation property of $A(\cdot)$ and (1.2), we obtain that

$$
\begin{aligned}
\left|\widehat{\sigma_{k}(\xi)}\right| & \leq\|b\|_{\infty} \int_{I_{k}}\left|\int_{\mathbb{R}} A(s)\left\{e^{-i \Phi(t) r\left(\xi^{\prime}\right)|\xi| s}-1\right\} d s\right| t^{-1} d t \\
& \leq C\|b\|_{\infty} \int_{I_{k}} t^{-1+d} r\left(\xi^{\prime}\right)|\xi| d t \leq C 2^{k d}\left|A_{\rho} \xi\right| .
\end{aligned}
$$

On the other hand, using Hölder's inequality, we have

$$
\left|\widehat{\sigma_{k}(\xi)}\right| \leq C\|b\|_{\infty} 2^{-k / 2} \mathcal{J}_{k}
$$

where

$$
\mathcal{J}_{k}=\left\{\int_{I_{k}}\left|\int_{\mathbb{R}} e^{-i \Phi(t) r\left(\xi^{\prime}\right)|\xi| s} A(s) d s\right|^{2} d t\right\}^{1 / 2} .
$$

To estimate $\mathcal{J}_{k}$, we choose a function $\psi \in C^{\infty}(\mathbb{R})$ satisfying

$$
\psi(t) \equiv 1 \quad \text { for }|t| \leq 1, \quad \psi(t) \equiv 0 \quad \text { for }|t| \geq 2 .
$$

Define $T_{k}$ by

$$
\left(T_{k} f\right)(t)=\chi_{I_{k}}(t) \int_{\mathbb{R}} e^{-i s \Phi(t) r\left(\xi^{\prime}\right)|\xi|} \psi(s) f(s) d s .
$$

Then

$$
T_{k} T_{k}^{*} f(t)=\int_{\mathbb{R}} L(t, s) f(s) d s
$$

where

$$
L(t, s)=\int_{\mathbb{R}} e^{i v(\Phi(s)-\Phi(t)) r\left(\xi^{\prime}\right)|\xi|} \psi^{2}(v) d v \chi_{I_{k}}(t) \chi_{I_{k}}(s) .
$$

We easily see that

$$
|L(t, s)| \leq C \chi_{I_{k}}(s) \chi_{I_{k}}(t) .
$$

On the other hand, by integration by parts, we have

$$
|L(t, s)| \leq C\left\{|\Phi(s)-\Phi(t)| r\left(\xi^{\prime}\right)|\xi|\right\}^{-1} \chi_{I_{k}}(t) \chi_{I_{k}}(s) .
$$

So

$$
|L(t, s)| \leq C\left\{|\Phi(s)-\Phi(t)| r\left(\xi^{\prime}\right)|\xi|\right\}^{-1 / 2} \chi_{I_{k}}(t) \chi_{I_{k}}(s) .
$$

Now by (1.3) we have

$$
|L(t, s)| \leq C 2^{k / 2}\left\{r\left(\xi^{\prime}\right)|\xi||s-t| 2^{d k}\right\}^{-1 / 2} \chi_{I_{k}}(s) \chi_{I_{k}}(t) .
$$

Therefore,

$$
\sup _{s>0} \int_{\mathbb{R}}|L(t, s)| d t \cong \sup _{t>0} \int_{\mathbb{R}}|L(t, s)| d s \cong 2^{k}\left(2^{d k} r\left(\xi^{\prime}\right)|\xi|\right)^{-1 / 2} .
$$


This shows

$$
\left\|T_{k} f\right\|_{2} \leq 2^{k / 2}\left(r\left(\xi^{\prime}\right)|\xi|\right)^{-1 / 4} 2^{-d k / 4}
$$

which leads to

$$
\left|\widehat{\sigma_{k}(\xi)}\right| \leq C\left(\left|A_{\rho} \xi\right| 2^{d k}\right)^{-1 / 4}
$$

By (3.5) and (3.6), we have

$$
\left|\widehat{\sigma_{k}(\xi)}\right| \leq C \min \left\{\left|2^{k d} A_{\rho} \xi\right|,\left|2^{k d} A_{\rho} \xi\right|^{-1 / 4}\right\} .
$$

Now (i) in Lemma (3.1) is proved. Thus to prove the theorem, by Lemma (3.1), it remains to show (ii) in Lemma 3.1.

By the method of rotation again we have

$$
\begin{aligned}
\widehat{\mu_{k}(\xi)} & =C \int_{2^{k} \leq|y| \leq 2^{k+1}}|y|^{-n}\left|a\left(y^{\prime}\right)\right| e^{-i \Phi(|y|)\left\langle y^{\prime}, \xi\right\rangle} d y \\
& =\int_{I_{k}} t^{-1} \int_{\mathbb{R}} G_{a}\left(s, \xi^{\prime}\right) e^{-i \Phi(t) s|\xi|} d s d t .
\end{aligned}
$$

By Lemma 2.1, we know $\operatorname{supp}\left(G_{a}\right)=\operatorname{supp}\left(F_{a}\right)=\left(\xi_{1}^{\prime}-2 r\left(\xi^{\prime}\right), \xi_{1}^{\prime}+2 r\left(\xi^{\prime}\right)\right)$. Define the measures $\left\{\lambda_{k}\right\}$ on $\mathbb{R}$ by

$$
\widehat{\lambda_{k}\left(\xi_{1}\right)}=\|a\|_{L^{1}\left(S^{n-1}\right)} \int_{I_{k}} t^{-1} e^{-i \Phi(t) \xi_{1}} d t .
$$

It is easy to see that, for each $k, \lambda_{k}$ is a positive measure. Also, by (1.2) and (1.3)

$$
\left|\widehat{\lambda}_{k}\left(\xi_{1}\right)-\widehat{\lambda}_{k}(0)\right| \leq C 2^{d k}\left|\xi_{1}\right|, \quad\left|\widehat{\lambda}_{k}\left(\xi_{1}\right)\right| \leq C\left|2^{d k} \xi_{1}\right|^{-1} .
$$

So by Theorem A of [DR], we know that $\sup _{k}\left|\lambda_{k} * h\right|$ is bounded in $L^{p}(\mathbb{R})$ for $1<p<\infty$. Let $\delta$ be the Dirac Delta function acting on $\left(x_{2}, \ldots, x_{n}\right)$. Then $\sup _{k}\left|\left(\lambda_{k} \otimes \delta\right) * f\right|$ is bounded in $L^{p}\left(\mathbb{R}^{n}\right)$.

Also we notice

$$
\left|\widehat{\mu_{k}(\xi)}-\widehat{\lambda_{k}\left(\xi_{1}\right)}\right| \leq C r\left(\xi^{\prime}\right)|\xi| 2^{d k}=C 2^{d k}\left|A_{\rho} \xi\right| .
$$

By the proof of (3.6), we also have

$$
\left|\widehat{\mu_{k}(\xi)}\right| \leq C\left|A_{\rho} \xi 2^{d k}\right|^{-1 / 4} .
$$

Choose the function $\Psi_{k}$ as in Lemma 2.3 and define, for each $k$,

$$
\nu_{k}(\xi)=\mu_{k}-\Psi_{k} *\left(\lambda_{k} \otimes \delta\right) .
$$

By (3.8) we have

$$
\begin{aligned}
\left|\widehat{\nu_{k}(\xi)}\right| & \leq \widehat{\mu_{k}(\xi)}-\widehat{\lambda_{k}(\xi)}\left|\widehat{\mid \lambda_{k}(\xi)}\right|\left|\widehat{\Psi}\left(2^{d k}\left|A_{\rho} \xi\right|\right)-1\right| \\
& \leq C\left|2^{d k} A_{\rho} \xi\right| .
\end{aligned}
$$

Therefore by (3.9) and (3.10) we easily see that

$$
\left|\widehat{\nu_{k}(\xi)}\right| \leq C \min \left\{\left|2^{d k} A_{\rho} \xi\right|,\left|2^{d k} A_{\rho} \xi\right|^{-1 / 4}\right\},
$$

where $\rho>0$ is the same as in (3.7).

By the definition of $\mu_{k}$, we have

$$
\sigma^{*} f \leq\left(\sum_{-\infty}^{\infty}\left|\nu_{k} * f\right|^{2}\right)^{1 / 2}+C M_{1} \cdots M_{n}\left(\sup _{k}\left(\lambda_{k} \otimes \delta\right) *|f|\right) .
$$


Now by Lemma 2.3 and (3.11), we find $\left\|\sigma^{*} f\right\|_{2} \leq C\|f\|_{2}$. Therefore, by Theorem $\mathrm{A}$ in $[\mathrm{DR}]$ and its proof (after a slight modification), we easily see $\left\|\sigma^{*} f\right\|_{p} \leq C\|f\|_{p}$ for all $1<p<\infty$. This proves the theorem.

Remark. In Theorem D, the condition $b \in L^{\infty}$ can be replaced by a weaker condition

$$
R^{-1} \int_{0}^{R}|b(t)|^{q} d t \leq C, \quad\left|\frac{1}{p}-\frac{1}{2}\right|<\min \left\{\frac{1}{2}, \frac{1}{q^{\prime}}\right\},
$$

where $C$ is a constant independent of $R>0$. Readers may see [Fa] for more details about this condition.

\section{REFERENCES}

[CZ1] A. P. Calderón and A. Zygmund, On the existence of certain singular integrals, Acta. Math. 88 (1952), 85-139. MR 14:637f

[CZ2] A. P. Calderón and A. Zygmund, On singular integrals, Amer. J. Math. 78 (1956), 289309. MR 18:894a

[Ch] L. Chen, On a singular integral, Studia Math., TLXXXV (1987), 61-72. MR 88i:42026

[Co] L. Colzani, Hardy Spaces on Sphere, Ph.D. Thesis, Washington University, St. Louis, MO, 1982.

[CTW] L. Colzani, M. Taibleson and G. Weiss, Maximal estimates for Cesàro and Riesz means on sphere, Indiana Univ. Math. J. 33 (1984), No. 6, 873-889. MR 86g:43012

[DR] J. Duoandikoetxea and J. L. Rubio de Francia, Maximal and singular integral operators via Fourier transform estimates, Invent. Math. 84 (1986), 541-561. MR 87f:42046

[Fa] D. Fan, Restriction theorems related to atoms, Ill. Jour. Math., Vol. 40, No. 1 (1996), 13-20. CMP 96:11

[Fe] R. Fefferman, A note on singular integrals, Proc. Amer. Math. Soc. 74 (1979), 266-270. MR 81e: 42025

[FP1] D. Fan and Y. Pan, Oscillatory integrals and atoms on the unit sphere, Manuscripta Math., 89 (1996), 179-192. CMP 96:07

[FP2] D. Fan and Y. Pan, $L^{2}$ boundedness of a singular integral operator, submitted.

[Na] J. Namazi, A Singular Integral, Ph.D. Thesis, Indiana University, Bloomington, 1984; Proc. Amer. Math. Soc. 96 (1986), 421-424. MR 87e:42023

[St] E. M. Stein, Problems in Harmonic Analysis Related to Curvature and Oscillatory Integrals, Proc. Inter. Cong. Math., Berkeley (1986), 196-221. MR 89d:42028

[SW] E. M. Stein and G. Weiss, Introduction to Fourier analysis on Euclidean spaces, Princeton Univ. Press, Princeton, NJ, 1971. MR 46:4102

Department of Mathematics, University of Wisconsin-Milwaukee, Milwaukee, WisCONSIN 53201

E-mail address: fan@alpha1.csd.uwm.edu

Department of Mathematics and Statistics, University of Pittsburgh, Pittsburgh, Pennsylvania 15260

E-mail address: yibiao@tomato.math.pitt.edu 